\title{
Increased Fatty Acid Oxidation in Differentiated Proximal Tubular Cells Surviving a Reversible Episode of Acute Kidney Injury
}

\author{
Aurélien Bataille ${ }^{a, b} \quad$ Pierre Galichon $^{a} \quad$ Nadjim Chelghoum ${ }^{d}$ \\ Badreddine Mohand Oumoussa ${ }^{c}$ Marie-Julia Ziliotis ${ }^{a}$ Iman Sadia ${ }^{a}$ \\ Sophie Vandermeerscha Noémie Simon-Tillauxa David Legouis ${ }^{a}$ \\ Raphaël Cohen $^{\mathrm{a}}$ Yi-Chun Xu-Dubois ${ }^{\mathrm{a}}$ Morgane Commereuc ${ }^{\mathrm{a}} \quad$ Eric Rondeau $^{\mathrm{a}, \mathrm{d}}$ \\ Stéphane Le Crom ${ }^{\mathrm{e}}$ Alexandre Hertiga
}

\begin{abstract}
aSorbonne Universités, INSERM UMR S 1155, Hôpital Tenon, Paris, France, bepartment of Anesthesiology and Critical Care Medicine, Lariboisiere Hospital, University Paris 7 Diderot, U942, Inserm, Paris, 'Sorbonne Universités, UPMC Université Paris 06, Inserm, UMS Omique, Plateforme P3S, Paris, dAPHP, Hôpital Tenon, Urgences Néphrologiques et Transplantation Rénale, 4 rue de la Chine, Paris, eSorbonne Universités, UPMC Université Paris 06, CNRS, Institut de Biologie Paris-Seine (IBPS), Evolution Paris Seine, Paris, France
\end{abstract}

\section{Key Words}

Acute Kidney Injury • Epithelium • Fibrosis • Chronic Kidney Disease • Fatty Acid Oxidation

\begin{abstract}
Background/Aims: Fatty acid oxidation (FAO), the main source of energy produced by tubular epithelial cells in the kidney, was found to be defective in tubulo-interstitial samples dissected out in kidney biopsies from patients with chronic kidney disease (CKD). Experimental data indicated that this decrease was a strong determinant of renal fibrogenesis, hence a focus for therapeutic interventions. Nevertheless, whether persistently differentiated renal tubules, surviving in a pro-fibrotic environment, also suffer from a decrease in $\mathrm{FAO}$, is currently unknown. Methods: To address this question, we isolated proximal tubules captured ex vivo on the basis of the expression of an intact brush border antigen (Prominin-1) in C57BL6/J mice subjected to a controlled, two-hit model of renal fibrosis (reversible ischemic acute kidney injury (AKI) or sham surgery, followed by angiotensin 2 administration). A transcriptomic high throughput sequencing was performed on total mRNA from these cells, and on whole kidneys. Results: In contrast to mice subjected to sham surgery, mice with a history of AKI displayed histologically more renal fibrosis when exposed to angiotensin 2. High throughput RNA sequencing, principal component analysis and clustering showed marked consistency within experimental groups. As expected, FAO transcripts were decreased in whole fibrotic kidneys. Surprisingly, however,
\end{abstract}


up- rather than down-regulation of metabolic pathways (oxidative phosphorylation, fatty acid metabolism, glycolysis, and PPAR signalling pathway) was a hallmark of the differentiated tubules captured from fibrotic kidneys. Immunofluorescence co-staining analysis confirmed that the expression of FAO enzymes was dependent of tubular trophicity. Conclusions: These data suggest that in differentiated proximal tubules energetic hyperactivity is promoted concurrently with organ fibrogenesis.

(C) 2018 The Author(s)

Published by S. Karger AG, Basel

\section{Introduction}

In recent years, data have accumulated suggesting that acute kidney injury (AKI) and chronic kidney disease (CKD) are interconnected [1]. Epidemiological databases, initially using diagnostic codes and then biological monitoring of patients treated for several conditions such as septic shock or cardiac surgery, combined to suggest a poor prognostic value for AKI, increasing the risk of CKD several-fold overall in the following months [2-4]. Surprisingly, this risk also exists in those experiencing a fully reversible episode of AKI [4, 5]. This has led to a new paradigm in the field of nephrology, that of "maladaptive repair" of the tubular epithelium, [6] as opposed to the traditional concept of restitutio ad integrum. At present, animal studies have established three major mechanisms in the tubular epithelium that explain a high rate of fibrogenesis in the aftermath of an AKI: i) the G2/M arrest of tubular epithelial cells, [7] ii) a partial epithelial to mesenchymal transition, [8, 9] and iii) the reduced capacity of epithelial cells to produce energy via fatty acid oxidation (FAO) [10]. All these mechanisms indicate potential targets for new drugs aiming to prevent AKI-induced CKD.

With respect to FAO, Hyun Mi Kang et al [10]. were the first to demonstrate that its defect plays a causal role in renal fibrogenesis. Importantly, this original discovery stemmed from the differential transcript analysis of tubulo-interstitial samples dissected out in kidney biopsies taken from control \pm hypertensive human patients vs. patients with CKD. By definition, sampling from CKD patients used fibrotic tissue. Experimental data were then collected using several animal models of kidney injury, including unilateral ureteral obstruction and folic acid nephropathy, that not only confirmed that FAO decreased in tubular epithelial cells subjected to an aggression, but also that this decrease was a strong determinant of renal fibrogenesis. This paper opened up the possibility of a new therapeutic AKI to delay the progression of CKD.

Here, we used a controlled, two-hit mouse model of renal injury in which we captured proximal tubules on the basis of the expression of Prominin-1, a brush border antigen, hence a marker of preserved differentiation. Interestingly, there was a correspondence between both next generation RNA sequencing of this specific population and immunofluorescence costaining analysis, suggesting that, in differentiated proximal tubules, energetic hyperactivity is promoted concurrently with organ fibrogenesis.

\section{Materials and Methods}

\section{Animal model}

Ethical and legal approval was obtained from the Comité d'éthique en expérimentation animale Charles Darwin N5 (French Ministry of Higher Education and Research \#00947.02). All C57Bl6/J mice were provided by Charles River (Wilmington, MA, USA). Surgery was performed in 8-week-old mice under thiopental and buprenorphine anesthesia, according to recommendations established to avoid variability [11] Blood samples were collected during isoflurane anesthesia. Angiotensin 2 (Sigma-Aldrich, Saint-Louis, MO, USA) was administered via osmotic subcutaneous mini-pumps (Model 2004, Alzet, Cupertino, CA, USA) also under thiopental anesthesia. During angiotensin 2 infusion, drinking water was enriched with $0.6 \%$ sodium chloride. 


\section{Cellular Physiology Cell Physiol Biochem 2018;47:1338-1351 \begin{tabular}{l|l} 
and Biochemistry & DOI: 10.1159/000490819 \\
Published onine: June 22, 2018 & $\begin{array}{l}\text { (c) } 2018 \text { The Author(s). Published by S. Karger AG, Basel } \\
\text { www.karger.com/cpb }\end{array}$
\end{tabular} \\ Bataille et al.: FAO in Injured Kidneys}

\section{Renal function}

Plasma urea and creatinine analysis was performed on a Konelab automate analyzer (Thermo Fisher Scientific, Waltham, MA, USA) using an enzymatic method.

\section{Histology}

Kidneys were fixed in $4 \%$ formaldehyde and cut in $4 \mu \mathrm{m}$ sections, for routine histological staining. Scanned images were obtained using an Olympus device (Tokyo, Japan). A specific Macro was programmed using the Image $\mathrm{J}^{\circledR}$ program to perform automatic quantification of fibrosis.

Quantitative reverse transcription polymerase chain reaction ( $q R T-P C R$ )

Total mRNA was extracted from mouse kidneys using miRNeasy Mini Kit (Qiagen, Hilden, Germany). After digestion by DNase 1, RNA was reverse transcribed using the Fermentas Maxima RT Kit (Thermo Fisher Scientific, Waltham, MA, USA) and amplified by PCR in a LightCycler 480 (Roche Diagnostics, Meylan, France) with SYBR Green (LightCycler 480 SYBR Green I Master; Roche Diagnostics) and specific primers for Hmgcs2 andFabp1. Glucuronidase (Gusb) and Actin b1 (Actb1) served as housekeeping genes. Primers were designed using the Universal Probe Library Roche website and are presented in Table 5. Quantification of gene expression was done according to the $2^{-\Delta \Delta C t}$ method.

\section{Library preparation and sequencing}

The total RNA concentration was measured with the NanoDrop1000 Spectrophotometer (Thermo Fisher Scientific, Waltham, MA, USA). RNA integrity was assessed using the Agilent 2100 Bioanalyzer RNA 6000 Nano Chip (Agilent Technologies, Santa Clara, CA, USA). The strand-specific libraries were prepared according to Sure Select Strand-Specific mRNA Library Preparation Protocol (Agilent Technologies, Santa Clara, CA, USA) starting from 500 ng of total RNA. Poly(A) RNA was purified from total RNA using oligo(dT) magnetic beads and then chemically fragmented at $94^{\circ} \mathrm{C}$ for $8 \mathrm{~min}$ to a size appropriate for RNA sequencing preparation. Cleaved RNA fragments were primed with random hexamers to synthesize first strand cDNA using transcriptase and random primers. To ensure strand specificity, a fresh Actinomycin D solution (Sigma-Aldrich, Saint-Louis, MO, USA) was prepared and mixed to First Strand Master Mix according to manufacturer's protocol. The double strand c-DNA synthesis was performed with incorporation of d'UTP instead of d'TTP in the second strand. The library construction was further processed from double strand cDNA fragments, which consisted of the following steps to ligate the necessary 5'and 3' specific adapters and incorporate indexes compatible with sequencing on Illumina platforms: End-repair, A-tailing, Adapter ligation, Size selection and PCR amplification. Previous to final amplification, the d'UTP marked strand was selectively degraded by Uracil-DNA-Glycosylase and the remaining strand was amplified to generate a cDNA library suitable for sequencing. The resulting library was then validated on Agilent 2100 Bioanalyzer DNA 1000 chip and quantified by Quant-iT dsDNA Broad range Assay (Thermo Fisher Scientific, Waltham, MA, USA). The fifteen barcoded libraries prepared were multiplexed and sequenced on two lanes of the HiSeq 1500 platform (2x100 pb, paired-end) (Illumina, San Diego, CA, USA).

\section{Bioinformatics analyses}

Raw data were first cleaned by excluding all quality control-failing reads and trimming bases from poor-quality ends of reads using fastq_illumina_filter_0.1 [12] and sickle_1.200 tools [13]. Reads were then aligned to the GRCm38 version of the mouse genome using the TopHat_2.0.11 program [14] and post-processed in order to remove all singletons, as well as fragments exceeding a defined size using samtools_0.1.18 [15].. These cleaned reads were finally used to quantify the expression level of genes and to perform the differential analysis using Cufflinks_2.2.1 [16] and CummeRbund_2.12.0 [17] software. For each of these steps a quality control was performed using numerous utilities such as FastQC_0.11.2, samstat-2.0, bamUtil_1.0.12 and R-3.2.2. Pathway analysis was performed using Lrpath [18, 19] and REVIGO, [20] available online.

\section{Results}

Course of renal fibrosis is dependent on a history of AKI after ischemia/reperfusion in mice We provide a model of AKI-related CKD where animals were subjected to ischemic tubular necrosis, given time to recover, and then challenged with a second and more chronic type of 


\section{Cellular Physiology Cell Physiol Biochem 2018;47:1338-1351 \begin{tabular}{ll|l} 
and & 10.1159/000490819 & $\begin{array}{l}\text { O 2018 The Author(s). Published by S. Karger AG, Basel } \\
\text { www.karger.com/cpb }\end{array}$ \\
\cline { 2 - 3 }
\end{tabular} \\ Bataille et al.: FAO in Injured Kidneys}

injury (i.e. angiotensin 2-induced hypertension) (Fig. 1A). A right nephrectomy and then a left renal ischemia (20-minute clamping of the pedicle (Fig. 1B)) transiently deteriorated the renal function of C57Bl6/J 8-week-old mice, as assessed by blood urea nitrogen and plasma creatinine, when compared to mice subjected to a right nephrectomy and sham surgery of the left kidney (Fig. 1A \& Fig. 2A). Mice experiencing AKI also displayed typical histological features of acute tubular necrosis (ATN), predominantly at the cortico-medullary junction: Giemsa staining revealed a well-defined area where cells were devoid of nuclei, and Periodic Acid Schiff staining showed evidence for loss of brush borders (Fig. 2B). At day 28, tissue repair was complete, as assessed by usual colorations. By that time, renal function was still slightly altered in the AKI group (Fig. 2A), but extracellular matrix deposition (assessed under a polarized microscope after Sirius red staining) was barely detectable (Fig. 2B). At this stage of resolved AKI i.e. day 28 post-AKI or sham surgery), $1 \mu$ g.kg-1 ${ }^{-1} \mathrm{~min}^{-1}$ of angiotensin 2 was continuously administered via s ubcutan e ous pumps and animal water was enriched with $0.6 \%$ sodium chloride to induce hypertension. Arterial pressure and heart rate $\mathrm{r}$ e s p o n d e d similarly in both groups (Fig. 3A). Mice were euthanized at day 56, to assess renal fibrosis. Twenty-eight days of angiotensin 2 resulted in i n t e r s t i ti a l fibrosis, albeit of significantly more intensity in the group of mice with a history of AKI (Fig. 3B), assessed by two blinded operators (Fig. 3C)

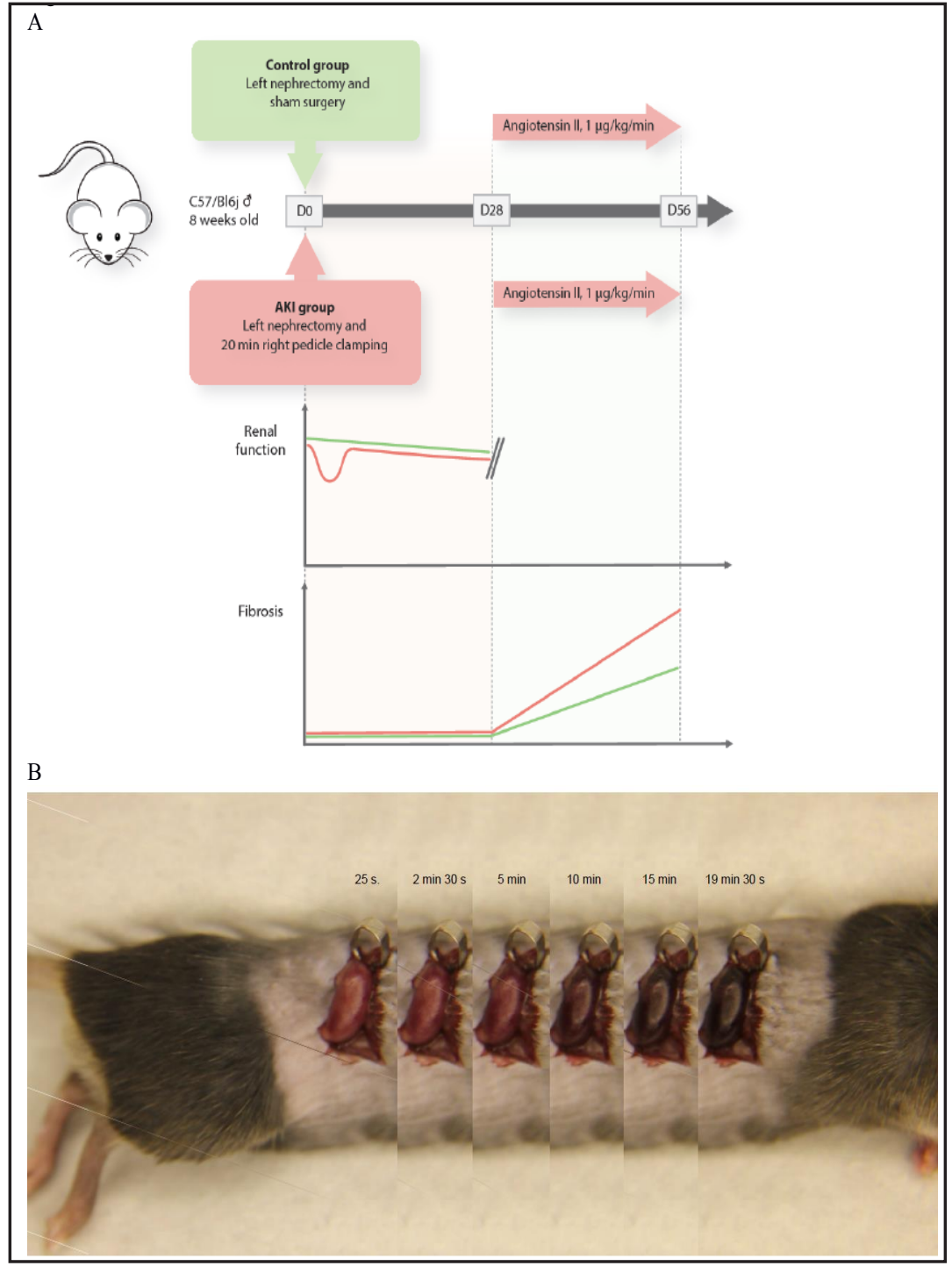

Fig. 1. Modeling chronic on acute injury in C57Bl6/j mice. (A) AKI-related model of CKD in adult ( $8 \mathrm{w} / \mathrm{o}$ ) male C57Bl6/j wild type mice. Left nephrectomy and subsequent, 20-min long ischemia-reperfusion injury of the right kidney cause a significant but reversible alteration of renal function with pristine kidneys by day 28. Subjected to a continuous infusion of angiotensin 2, mice develop hypertension, and significantly more fibrosis than mice with a history of left nephrectomy plus sham surgery of the right kidney. (B) Ischemia-reperfusion model 20 minutes of renal ischemia was performed using right pedicle clamping. Decoloration and recoloration of the kidney was closely monitored. 


\section{Cellular Physiology Cell Physiol Biochem 2018;47:1338-1351 \begin{tabular}{l|l} 
DOI: 10.1159/000490819 & $\begin{array}{l}\text { O 2018 The Author(s). Published by S. Karger AG, Basel } \\
\text { www.karger.com/cpb }\end{array}$
\end{tabular}

and an automated m or phologi c a l technique (Fig. 3D), with good correlation between techniques (Fig. 3E). At this time point, plasma urea and creatinine were similar between groups (Fig. 3F). Of note, in mice with or without a history of AKI but not subjected to a second hit, fibrosis at day 56 was comparably mild $(p=0.1355)$. Overall, this controlled mouse model of A KI - d e p e ndent, a n g i o t e n s i n 2-induced interstitial fibrosis may be used to study the tubular epithelial program placed under a common stress (hypertension) following successful renal repair, and as such is relevant to human disease.

\section{Transcriptome of} proximal tubular cell analysis after kidney injury

In each mouse group we isolated ex vivo the fraction of proximal tubules which were persistently differentiated, according to a technique we have previously published [21]. In brief, this technique profits from the expression of Prominin-1 in the brush border of proximal tubular cells. We isolated cells at three different time points (Fig. 4A): the right kidney removed at day 0 (control, $n=3$ ), the left kidney from mice euthanized at day 28 post-AKI (AKI, $n=3$ ) or post-sham surgery (Sham, $\mathrm{n}=3$ ), and the left kidney from mice subjected to 28 days of angiotensin 2 after a reversible AKI (AKI-angio2, $n=3$ ) or after a sham surgery (Sham-angio2, $n=3$ ). The procedure resulted in a $81-87 \%$ pure population of Prominin- $1^{\text {high }}$ cells (Table 1 ), and allowed for the isolation of high-quality RNA (i.e. appropriate for high throughput sequencing (Table 2)). Biological samples were processed using Illumina ${ }^{\odot}$ RNA-seq technology. A mean of 46 million reads were generated for each sample and aligned to the mouse reference genome. This mapping 


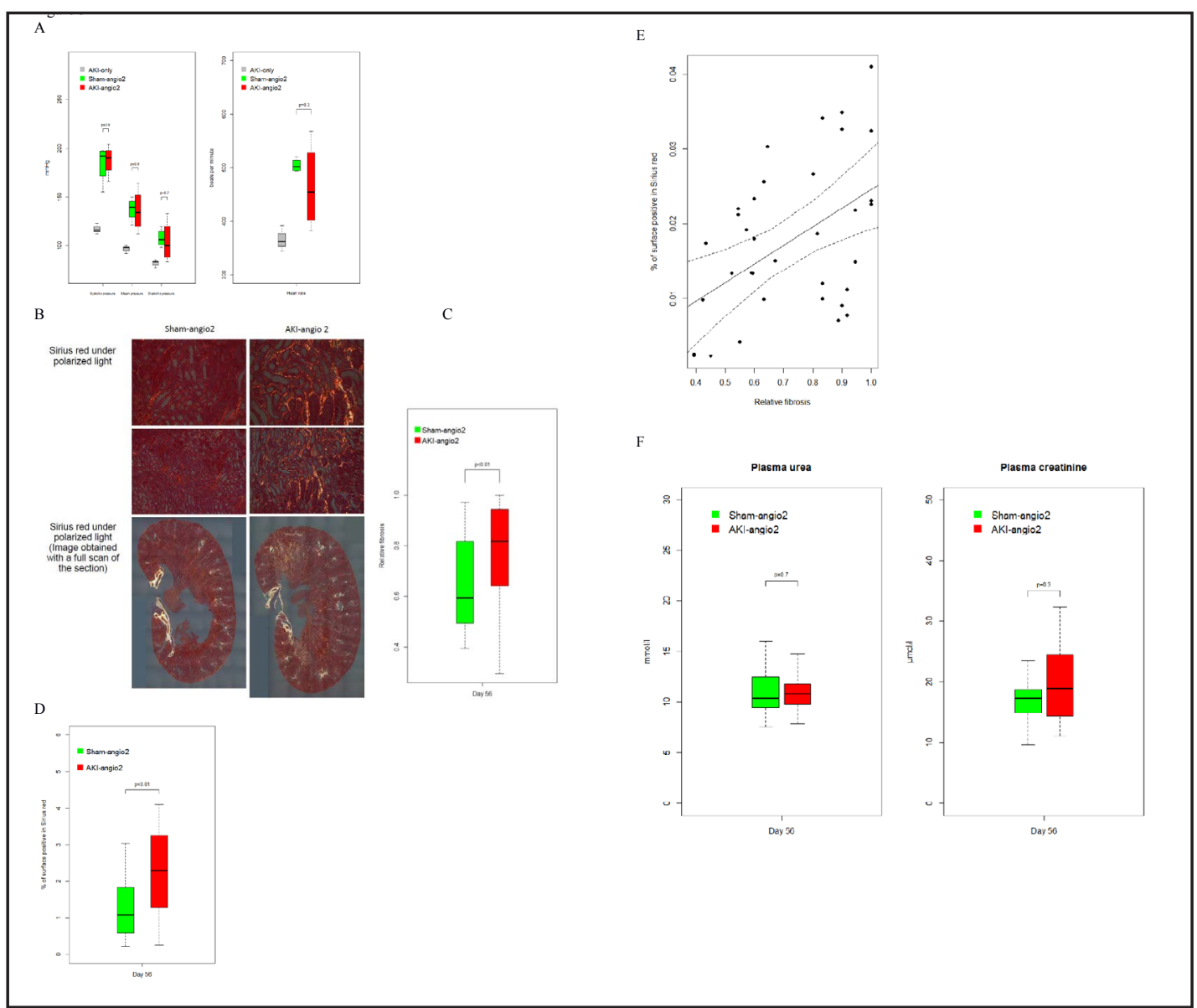

Fig. 3. Fibrosis intensity after 28 days under angiotensin 2 is determined by a history of resolving AKI. (A) Blood pressure and heart rate were measured invasively in different groups at day 56 . A control group with a past history of ischemic AKI but without administration of angiotensin 2 is added. No difference between AKI-angio2 and Sham-angio2 groups was noted. Data were obtained from 4 mice in the AKI-angio2 and Sham-angio2 group and from 3 mice in the AKI-only group. (B) Histological comparison of fibrosis between Sham-angio2 and AKI-angio2 groups. Sirius red staining was used under polarized light microscopy. Different magnifications are shown (20x and 40x). Data were obtained from 4 different experiments, each including at least 5 mice per group. (C) Comparison of extracellular collagen deposition at day 56 in the AKI-angio2 and Sham-angio2 groups, by polarization light microscopy, assessed by two blinded operators. A significant increase in fibrosis was observed in AKI-angio2 mice when compared to Sham-angio2 mice. (D) Automatic evaluation of fibrosis using Image J. Quantitative evaluation of fibrosis was performed using a specifically designed Image J macro, using kidney sections analyzed under polarized microscopy. Data obtained from 3 different experiments, each including at least 5 mice per group. (E) Correlation between manual and automatic evaluation of fibrosis. Solid line: regression line; dashed line: 95\% confidence intervals. Data obtained from 3 different experiments, each including at least 5 mice per group. (F) Plasma urea and creatinine at day 56 in AKI or Sham surgery mice. Data were obtained from 4 different experiments, each including at least 5 mice per group.

was possible for $90 \%$ of the reads, out of which $95 \%$ had a high quality score (certainty $>0.999$ ). Overall similarity between samples belonging to the same experimental group was assessed by principal component analysis (PCA) (Fig. 4B \& Fig. 4C).

Comparing the AKI group to the Sham group gave the following results: at day 28 , among the 548 genes differentially regulated, 301 were up-regulated ( 8 more than 3-fold) and 247 were down-regulated (4 more than 3-fold) (Fig. 4D \& Table 3). At day 56, i.e. after 28 additional days of angiotensin 2, 1258 genes were differentially regulated. A history of AKI was 
Table 1. Data obtained on samples used to confirm correct Prominin-1 $1^{\text {high }}$ cells isolation from flow cytometry, using anti-Prominin 1 antibody coupled to Allophycocyanin and a Hoechst gating to detect intact cells

\begin{tabular}{|c|c|c|c|c|}
\hline & $\begin{array}{l}\text { Total number of cells in } \\
\text { dissociated kidney }\end{array}$ & $\begin{array}{l}\% \text { of Prominin- } 1 \text { high cells in } \\
\text { dissociated kidney }\end{array}$ & $\begin{array}{l}\text { Total number of cells in } \\
\text { isolated fraction }\end{array}$ & $\begin{array}{c}\% \text { of Prominin-1 high cells in } \\
\text { isolated fraction }\end{array}$ \\
\hline Control & 5820000 & 13.30 & 726000 & 82.86 \\
\hline Control & 7020000 & 12.48 & 673000 & 83.15 \\
\hline Control & 9260000 & 13.87 & 689000 & 85.56 \\
\hline Sham & 15820000 & 9.36 & 1050000 & 80.69 \\
\hline Sham & 9360000 & 14.06 & 972000 & 86.16 \\
\hline Sham & 11460000 & 18.55 & 1290000 & 88.69 \\
\hline AKI & 8240000 & 15.52 & 975000 & 81.25 \\
\hline AKI & 8640000 & 18.40 & 722000 & 88.00 \\
\hline AKI & 9080000 & 17.89 & 787000 & 87.13 \\
\hline $\begin{array}{l}\text { Sham- } \\
\text { angio2 }\end{array}$ & 11240000 & 7.21 & 310000 & 84.63 \\
\hline $\begin{array}{l}\text { Sham- } \\
\text { angio2 }\end{array}$ & 10769000 & 11.56 & 597000 & 87.46 \\
\hline $\begin{array}{l}\text { Sham- } \\
\text { angio2 }\end{array}$ & 7560000 & 14.28 & 721000 & 85.25 \\
\hline $\begin{array}{l}\text { AKI- } \\
\text { angio2 }\end{array}$ & 8520000 & 11.67 & 416000 & 87.79 \\
\hline $\begin{array}{l}\text { AKI- } \\
\text { angio2 }\end{array}$ & 10320000 & 14.21 & 767000 & 86.59 \\
\hline $\begin{array}{l}\text { AKI- } \\
\text { angio2 }\end{array}$ & 9500000 & 14.46 & 587000 & 84.54 \\
\hline
\end{tabular}

associated with the up-regulation of 502 genes (among which 21 were more than 3 -fold), and down-regulation of 756 genes (among which 29 genes reached 3 -fold variation) (Fig. 4D \& Table 4]. Remarkably, dendrogram analysis (Fig. 4E \& Fig. 4F) revealed that sham-operated mice and mice with a history of AKI 28 days earlier were transcriptionally close. More variation was seen in mice exposed to angiotensin 2 , whether or not mice had a history of AKI. PCA then singularized three groups of mice: 1) day 0 control mice, 2) day 28 Sham, day $28 \mathrm{AKI}$, and 3) day $56 \mathrm{AKI}$-angio2, day 56 Sham-angio2 mice (Fig. 4B \& Fig. 4C). Strikingly, mice at day 28 were close, with a greater difference between AKI-angio 2 and Sham-angio2 mice than between $\mathrm{AKI}$ and Sham mice.

\section{Molecular pathways associated with a history of ischemic AKI}

A gene pathway analysis was performed to identify biological pathways differentially regulated in mice with/without a history of resolving AKI and subjected to a second 'hit'. Paradoxically, this analysis highlighted metabolic pathways as up-regulated (metabolic pathways, fatty acid metabolism, oxidative phosphorylation, glycolysis/gluconeogenesis, PPAR signaling pathway, peroxisome) and
Table 2. Data obtained from BioAnalyzer, (Agilent Technologies, Santa Clara, CA, USA) on samples after RNA isolation from Prominin ${ }^{\text {high }}$ cells using miRNeasy Mini Kit (Qiagen, Hilden, Germany)

\begin{tabular}{ccc}
\hline RNA concentration $(\mathrm{ng} / \mu \mathrm{l})$ & rRNA Ratio $[28 \mathrm{~s} / 18 \mathrm{~s}]$ & RNA Integrity Number \\
\hline 116 & 1.7 & 8.6 \\
89 & 1.4 & 8.3 \\
105 & 1.8 & 8.7 \\
175 & 1.3 & 8.1 \\
164 & 1.2 & 8.2 \\
225 & 1.4 & 8.2 \\
170 & 1.8 & 8.4 \\
170 & 1.4 & 8.2 \\
219 & 1.4 & 8.1 \\
79 & 1.4 & 8.0 \\
103 & 1.3 & 8.2 \\
35 & 1.3 & 8.3 \\
68 & 1.2 & 7.9 \\
120 & 1.3 & 8.0 \\
108 & 1.4 & 8.0 \\
\hline
\end{tabular}

Table 3. Comparing groups at day 28, 8 genes were up-regulated more than 3 fold and 4 were down- regulated more than 3 fold in the AKI group compared to the Sham group

\begin{tabular}{lccc}
\hline Gene name & Log2 fold change & $\begin{array}{c}\text { Gene } \\
\text { name }\end{array}$ & Log2 fold change \\
\hline Col3a1 & 2.99951 & Zbtb16 & -1.85055 \\
Cd200 & 2.30715 & Cish & -2.84367 \\
Bc16 & 1.91868 & Hspa1a & -3.48842 \\
Irx2 & 1.78838 & Hspa1b & -3.88204 \\
Hsd11b2 & 1.77242 & & \\
AI838599 & 1.76239 & & \\
Pla1a & 1.64685 & & \\
Ighm & 1.64164 & & \\
\hline
\end{tabular}


Fig. 4. Transcriptome analysis of proximal tubular cells in the context of organ fibrosis triggered by acute kidney injury. (A) Number of mice subjected to Prominin- $1^{\text {high }}$ tubular cells ex vivo analysis. 3 mice per group were included at 3 different time points: at day 0 (left kidneys), at day 28 (right kidney from AKI and Sham groups), and at day 56 (after 28 days of angiotensin 2). (B) Principal component analysis of each of the 15 mice. Principal component 1 and 2 are plotted here. Mice subjected to AKI are identified as "IR". Angioten$\sin 2$ is indicated as "Angio2". (C) Principal component analysis of each of the 15 mice. Principal component 2 and 3 are ploted (Please note that mice from the AKI group are identified as IR and mice from AKI-angio2 group are identified as IR-angio2). There was a good separation in between Control, day 28 and day 56 mice, with more difference in between AKI-angio2 and Sham-angio2 mice than AKI and Sham mice. (D) Genes significantly differently up- or down-regulated by the analysis. In the red-orange scale, genes significantly up-regulated in between groups (e.g. 2443 genes were significantly upregulated in the AKI-angio2 group when compared to the Control group). In the dark blue-blue scale, genes significantly down-regulated between groups (e.g. 2484 genes were significantly down-regulated in the AKI-angio2 group when compared to the Control group). (E) Gene clustering of each of the 15 mice. Each group has been correctly identified except one Shamangio2 mouse, which was closer to the group of mice euthanized at day 28. (F) Gene clustering of the 5 mouse groups.

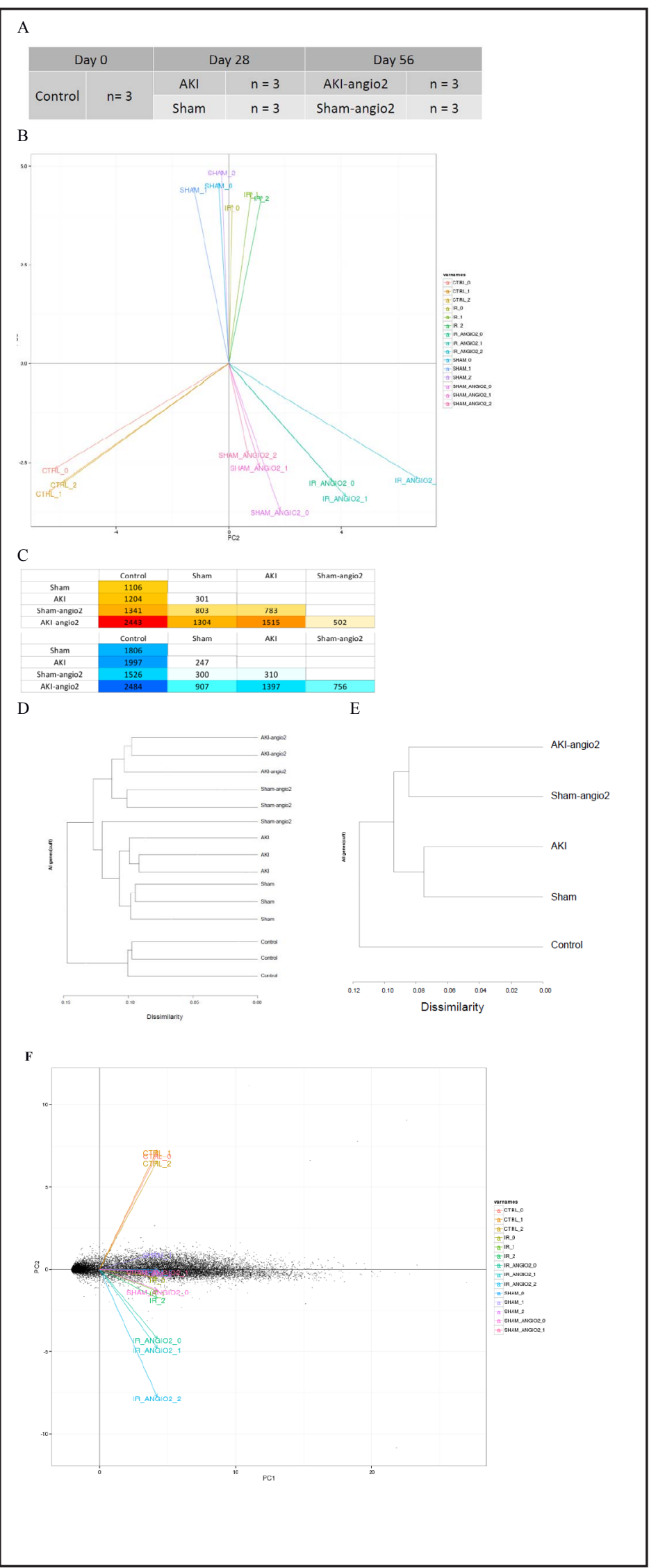


pro-fibrotic pathways as down-regulated (TGF-beta signaling pathway and Wnt signaling pathway) (Fig. 5A \& Fig. 5B). Only the PPAR signaling pathway was already detectable at day 28 post-AKI (data not shown).

Identification of Prominin-1 positive tubular cells up-regulating metabolic enzymes Hmgcs2 and Fabp1

We focused on two genes 3-hydroxy-3-methylglutaryl-Coenzyme A synthase 2 (Hmgcs 2 ) and fatty acid binding protein 1 (Fabp1) - specifically up-regulated at the transcriptional level in mice with a history of AKI (Fig. 6A \& Fig. 6B) because they come into play at the two extremes of lipid metabolism. Fabp1 plays a role in fatty acid transport and uptake and Hmgcs2 catalyzes the first reaction of ketogenesis after lipid oxidation. While PCR performed on whole kidney extracts showed a down-regulation of these transcripts throughout our model as expected (Fig. 6C), opposite results were obtained in Prominin- $1^{\text {high }}$ cells (Fig. 6B).Using immunofluorescence in whole kidney sections we observed that Prominin-1 expression faded with progression of tubular atrophy (Fig. 6D) while Hmgcs2 was up-regulated in proximal tubules from mice exposed to angiotensin 2 (Fig. $6 \mathrm{E}$ ), suggesting metabolic hyperactivity of differentiated proximal tubular cells facing a second insult.

\section{Discussion}

In mammalian tubular epithelial cells from kidneys recovering from injury, an altered capacity to synthesize the enzymes required for the oxidation of fatty acids, a major source of energy, is causally associated with renal fibrogenesis. The present animal study adds granularity to the understanding of the metabolic phenotype of proximal tubules existing in a fibrotic environment. Our data thus indicate that, in contrast to the metabolic signature of the kidney as a whole organ, where FAO is globally decreased - in atrophic tubules in particular - the signature of preserved (Prominin-1 ${ }^{\text {high }}$ ) proximal tubules is dramatically different. Here, while metabolic enzymes are poised for activation, pro-fibrotic genes are silent, FAO being the most enriched gene pathway when comparing hypertensive mice with or without interstitial fibrosis.

Table 4. Comparing groups at day 56, 21were up-regulated more than 3 fold and 29 were down- regulated more than 3 fold in the AKI-angio2 group compared to the Sham-angio2 group

\begin{tabular}{|c|c|c|c|}
\hline Gene name & $\log 2$ fold & Gene name & $\log 2$ \\
\hline Tmem254a & Inf & Kantr & - \\
\hline Hmgcs2 & 3.72207 & Hspa1b & - \\
\hline Cbr3 & 3.25058 & Rpl30 & - \\
\hline Zbtb16 & 2.79495 & Hspa1a & - \\
\hline Aqp2 & 2.70909 & Ifi 47 & - \\
\hline Fabp1 & 2.5585 & Olfr56 & - \\
\hline Сур4a14 & 2.51887 & Ppia & - \\
\hline Rarres2 & 2.37899 & Gm11407 & - \\
\hline Hist1h2bg & 2.17734 & Derl3 & - \\
\hline Apela & 2.11269 & Gm6977 & - \\
\hline Trim60 & 2.11269 & Cxcl10 & - \\
\hline Foxq1 & 2.0027 & Gm13202 & - \\
\hline$A \cot 1$ & 1.78963 & Gbp10 & - \\
\hline Slc5a10 & 1.77876 & Gbp6 & - \\
\hline $1190002 \mathrm{~F} 15 \mathrm{Ri}$ & 1.77807 & Gm12185 & - \\
\hline Pcsk6 & 1.71984 & Tgtp1 & - \\
\hline Сур4а10 & 1.71379 & Gm13675 & - \\
\hline Chrdl2 & 1.70973 & Fos & - \\
\hline Krt19 & 1.65688 & Iigp 1 & - \\
\hline $\operatorname{Lcn} 2$ & 1.62058 & Ighm & - \\
\hline \multirow[t]{9}{*}{ Clic6 } & 1.61823 & BC023105 & - \\
\hline & & Gm27875 & - \\
\hline & & $J p x$ & - \\
\hline & & Gm11408 & - \\
\hline & & Gm11410 & -3.663 \\
\hline & & Egr1 & - \\
\hline & & St7 & - \\
\hline & & Gm9763 & $-\operatorname{Inf}$ \\
\hline & & Gm4462 & $-\operatorname{Inf}$ \\
\hline
\end{tabular}

Table 5. Primers for mRNA quantitative RT-PCR

\begin{tabular}{lrr}
\hline $\begin{array}{l}\text { Tested } \\
\text { genes }\end{array}$ & Sense & Antisense \\
\hline Hmgcs2 & 5'-CTGTGGCAATGCTGATCG-3' & 5'-TCCATGTGAGTTCCCCTCA-3' \\
Fabp1 & 5'-CCATGACTGGGGAAAAAGTC-3' & 5'-GCCTTTGAAAGTTGTCACCAT-3' \\
Gusb & 5'-CTCTGGTGGCCTTACCTGAT-3' & 5'-CAGTTGTTGTCACCTTCACCTC-3' \\
Actb & 5'-CTAAGGCCAACCGTGAAAAG-3' & 5'-ACCAGAGGCATACAGGGACA-3' \\
\hline
\end{tabular}




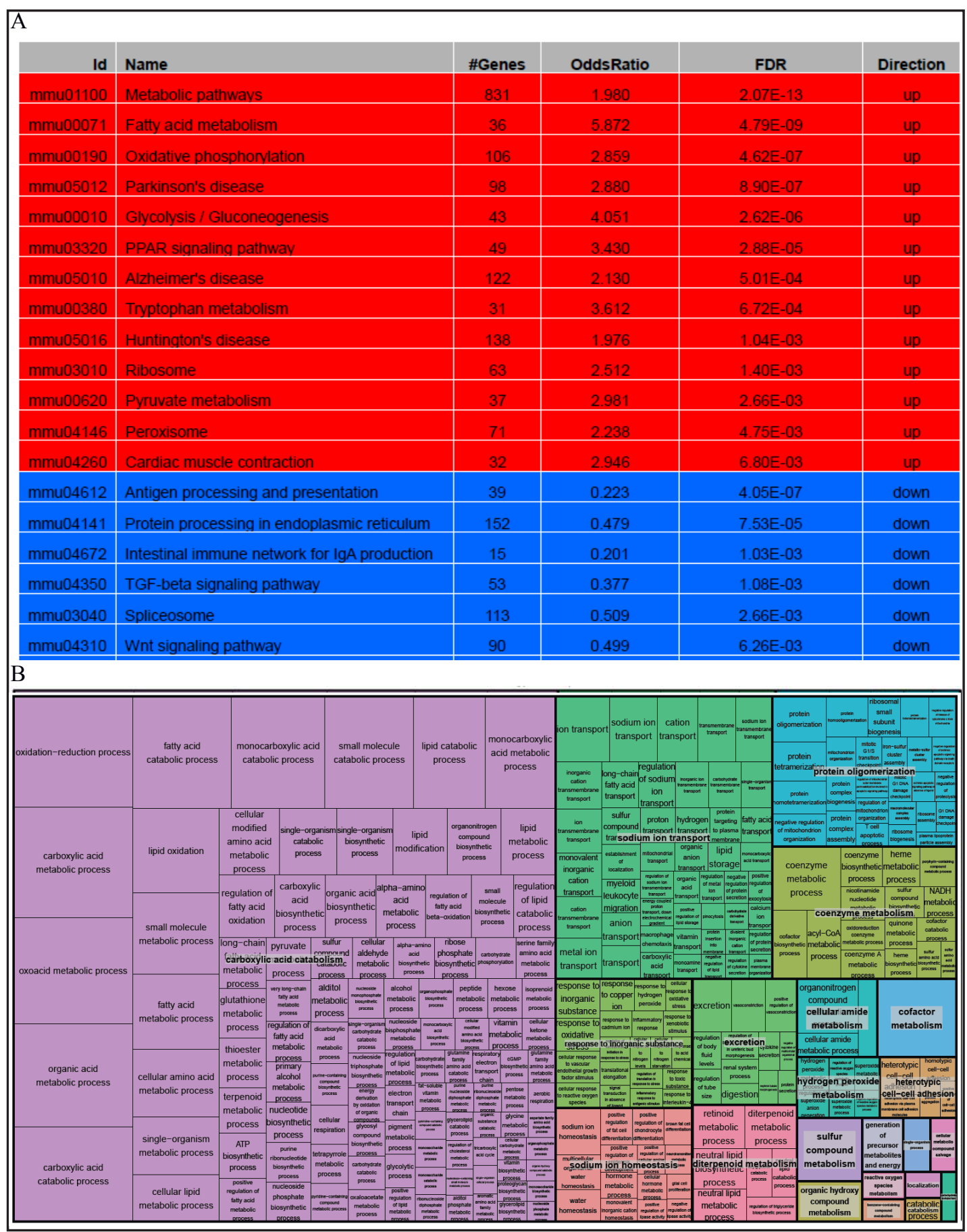

Fig. 5. Cell pathways regulation given by the transcriptome analysis. (A) Pathway differentially regulated comparing AKI-angio2 and Sham-angio2 groups. A false discovery rate (FDR) cut-off of 0.01 was used to select pathways. In red, pathways up-regulated in AKI-angio2 vs Sham-angio2; in blue, pathways downregulated. (B) REVIGO visualization of Gene Ontology Biological Pathways up-regulated in the AKI-angio2 group compared to the Sham-angio2 group. Main signal was towards up-regulation of metabolism and oxidative phosphorylation.

At first sight, these results might seem contradictory to those reported previously in the literature [10]. However, the pro-fibrotic role of the down-regulation of fatty acid metabolism was discovered by dissecting out atrophic tubules in fibrotic kidneys. In contrast, we 


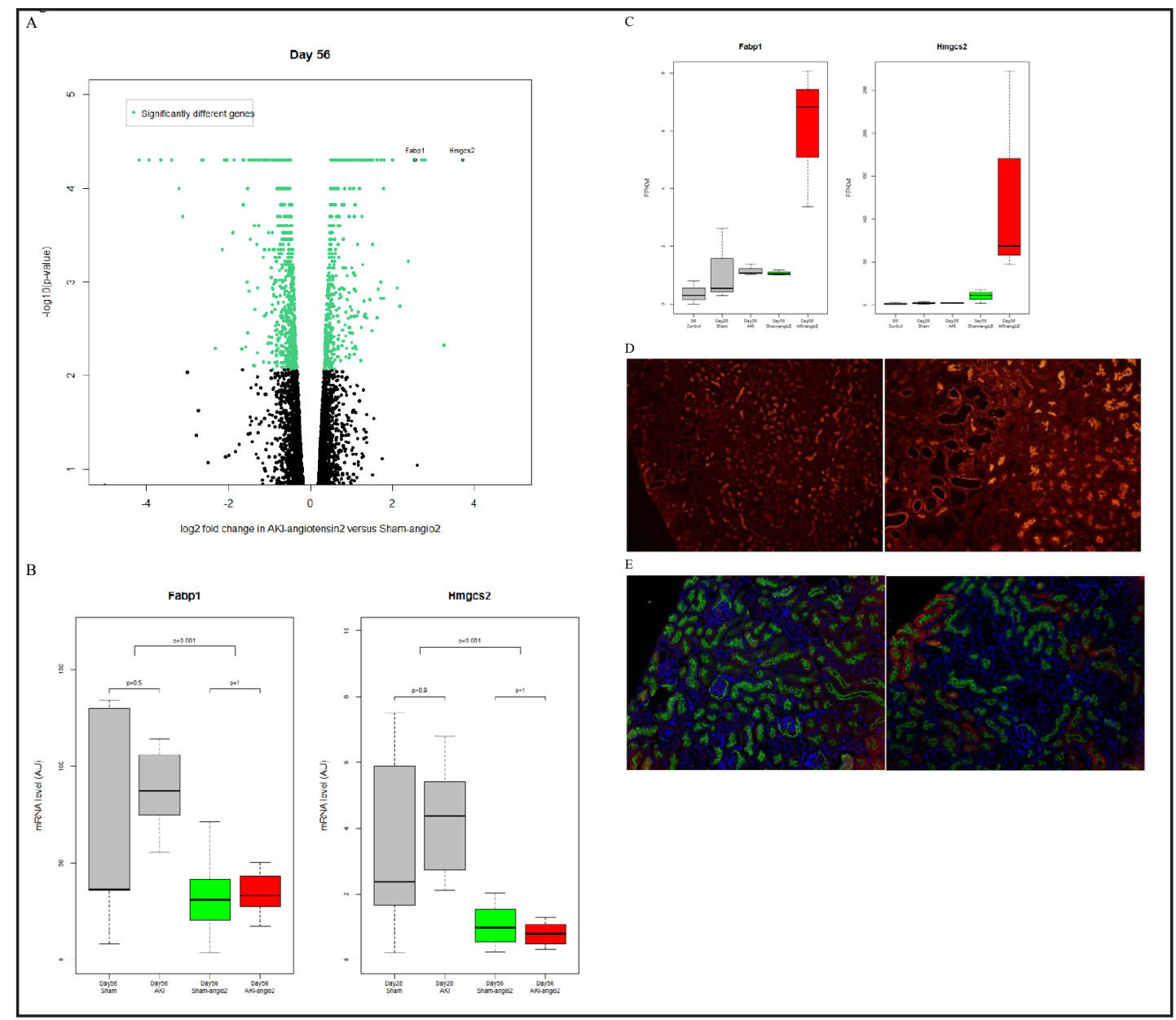

Fig. 6. High expression of metabolic enzymes in Prominin-1+ cells. (A) Volcano plot of AKI-angio2 and Sham-Angio2 gene expression. Up-regulation of FABP1 and HMGSC2 is shown in Prominin-1 ${ }^{\text {high }}$ cells in the AKI-angio2 group. (B) mRNA expression level of Fabp1 and Hmgcs2 in total kidney normalized to housekeeping genes (Actb and Gusb). (AU : arbitrary unit). (C) Fabp1 and Hmgcs2 in Prominin-1 $1^{\text {high }}$ cells: individual values given by transcriptome analysis. (FPKM: fragments per kilobase of exon per million fragments mapped). (D) Immunofluorescence of Prominin-1 at day 56 in a fibrotic kidney. Different magnifications are shown: 20x (right) and 40x (left). (E) Co-localization of HMGCS2 (red) with megalin (green) and DAPI (blue), here used as a marker of Prominin- $1^{\text {high }}$ proximal tubular cells at day 56 in a normal (right) and fibrotic kidney (left). Original magnification 20x.

captured tubules that exist in a fibrotic environment but still express a differentiation marker, Prominin-1. In addition, the report by Kang et al [10]. that etoxomir, an agent that further inhibits FAO, has a pro-fibrotic effect, necessarily implies that this metabolic pathway is still active in some cells in fibrotic kidney. These results have been confirmed elsewhere [22]. Our results identify Prominin- $1^{\text {high }}$ proximal tubules as cells that are, indeed, metabolically hyperactive. The long-term consequences of this hypermetabolic state are unknown, either at the cellular or whole organ level.

The differential evolution of epithelial cell sub-populations is one of the discoveries of our approach. It is in line with results from the literature that may seem contradictory. We could consider a heterogeneity of epithelial populations in response to aggression and that there is a kind of metabolic switch that explains the differences highlighted by our study. Cell signalling of mitogen-activated protein kinases (MAPKs) is illustrative [23]. MAPKs are differentially involved in renal responses to hypoxic/ischemic stress. They provide signals of growth, proliferation and cell differentiation, but also apoptosis and fibrosis. 


\section{Cellular Physiology Cell Physiol Biochem 2018;47:1338-1351

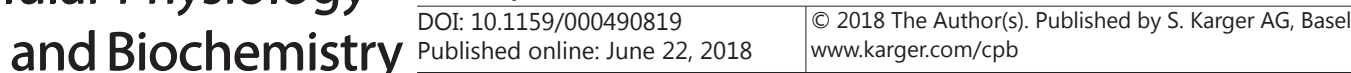 \\ Bataille et al.: FAO in Injured Kidneys}

At the organ level, the mechanisms of cellular adaptation and repair operate from the ischemic phase [24]. Ischemia-reperfusion causes cell apoptosis that can be reduced by pharmacological agents [25]. A better understanding of the kinetic mechanisms of survival and repair and metabolic adaptations could allow to better influence the cell fate.

Cell selection using a surface protein is an important step in our approach. There are indeed phenotypic changes in tubular cells and the expression of surface molecules including transporters is well documented. For instance, the decline in the expression of Organic Anion transporter 1 and 3 is transcriptionally mediated by COX1 metabolites after ischemia [26]. If it appears that the uremia is an influence on the expression of these epithelial proteins, [27] in our case, it is indeed a consequence of the first ischemic hit, because the levels of uremia are relatively low. We remain nevertheless dependent on the model developed here. We do not address here the potential link between metabolic hyperactivity and tubular fate in general, and this is one limit of our study. Understanding the biological mechanisms, and the long-term consequences of this peculiar program, will undoubtedly require further exploration.

Another interesting aspect of our study is that, by taking an unbiased approach, we have consolidated the recent theories that even a functionally and histologically reversible episode of ischemic AKI has long-term consequences, and sensitizes the kidney to an accelerated fibrotic process if a second hit occurs. Not only did the PCA correctly assemble the mice according to their history, but it also revealed that proximal tubular cells from mice exposed to angiotensin 2 have a different program, depending on whether they have a history of AKI or of sham surgery.

Our results are consistent with therapeutic lines recently raised. Mitochondrial dysfunction secondary to ischemia-reperfusion accounts for the metabolic dysfunction. A protective action to limit the oxidative stress or to limit the mitochondrial susceptibility in healthy hypermetabolic differentiated cells could be effective: nitro-arachidonic acid administration before angiotensin II-induced mitochondrial dysfunction has participated to less cell damage [28] and a specific rescue action could be done cells with FAO dysfunction: silymarin improves oxidative stress and preserve mitochondrial dysfunction by preventing accumulation of lipids in the kidney [29].

\section{Abbreviations}

AKI (acute kidney injury); CKD (chronic kidney disease); ATN (acute tubular necrosis); PCA (principal component analysis); Hmgcs2 (3-hydroxy-3-methylglutaryl-Coenzyme A synthase 2); Fabp1 (fatty acid binding protein 1).

\section{Acknowledgements}

The authors thank Ms Caroline Martin and her team for animal care. Aurélien Bataille received a grant from the French Society of Anesthesiology and Intensive Care (SFAR) in 2013 and a salary grant from the French Institute for Medical Research (Inserm, 2014-2016). Alexandre Hertig received an INTERFACE grant from Inserm. This project received academic grants from the Fondation pour la Recherche Médicale (FRM), and the French Society of Nephrology, Dialysis, and Transplantation (SFNDT). Financial support was obtained from Astellas Pharma, and Behring.

AB conducted most of the experiments and analyzed the results. PG, MJZ, IS, NS, DL, $\mathrm{RC}$ and MC participated in the ex vivo capture of proximal tubules, and analyzed the results. SV conducted the real-ime PCR experiments and analyzed the results. YC-XD performed co-staining experiments. NC, BMO, and SL conducted NGS sequencing of the samples, and analyzed the results. ER contributed to the analysis of the results. AH conceived the idea for the project, and wrote most of the paper.

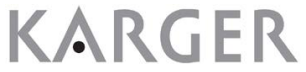




\section{Cellular Physiology Cell Physiol Biochem 2018;47:1338-1351 \begin{tabular}{l|l} 
DOI: 10.1159/000490819 & $\begin{array}{l}\text { O 2018 The Author(s). Published by S. Karger AG, Basel } \\
\text { www.karger.com/cpb }\end{array}$ \\
\hline
\end{tabular} \\ Bataille et al.: FAO in Injured Kidneys}

\section{Disclosure Statement}

The authors declare that they have no conflicts of interest with the contents of this article.

\section{References}

1 Chawla LS, Eggers PW, Star RA, Kimmel PL: Acute kidney injury and chronic kidney disease as interconnected syndromes. N Engl J Med 2014;371:58-66.

- Wald R, Quinn RR, Luo J, Li P, Scales DC, Mamdani MM, Ray JG, University of Toronto Acute Kidney Injury Research Group: Chronic dialysis and death among survivors of acute kidney injury requiring dialysis. JAMA 2009;302:1179-1185.

- I Ishani A, Xue JL, Himmelfarb J, Eggers PW, Kimmel PL, Molitoris BA, Collins AJ: Acute kidney injury increases risk of ESRD among elderly. J Am Soc Nephrol 2009;20:223-228.

4 Bucaloiu ID, Kirchner HL, Norfolk ER, Hartle JE, Perkins RM: Increased risk of death and de novo chronic kidney disease following reversible acute kidney injury. Kidney Int 2012;81:477-485.

5 Legouis D, Galichon P, Bataille A, Chevret S, Provenchère S, Boutten A, Buklas D, Fellahi JL, Hanouz JL, Hertig A: Rapid Occurrence of Chronic Kidney Disease in Patients Experiencing Reversible Acute Kidney Injury after Cardiac Surgery. Anesthesiology 2017;126:39-46.

-6 Bonventre JV, Yang L: Cellular pathophysiology of ischemic acute kidney injury. J Clin Invest 2011;121:4210-4221.

7 Yang L, Besschetnova TY, Brooks CR, Shah JV, Bonventre JV: Epithelial cell cycle arrest in G2/M mediates kidney fibrosis after injury. Nat Med 2010;16:535-543, 1p following 143.

> Grande MT, Sánchez-Laorden B, López-Blau C, De Frutos CA, Boutet A, Arévalo M, Rowe RG, Weiss SJ, LópezNovoa JM, Nieto MA.: Snail1-induced partial epithelial-to-mesenchymal transition drives renal fibrosis in mice and can be targeted to reverse established disease. Nat Med 2015;21:989-997.

-9 Lovisa S, LeBleu VS, Tampe B, Sugimoto H, Vadnagara K, Carstens JL, Wu CC, Hagos Y, Burckhardt BC, Pentcheva-Hoang T, Nischal H, Allison JP, Zeisberg M, Kalluri R: Epithelial-to-mesenchymal transition induces cell cycle arrest and parenchymal damage in renal fibrosis. Nat Med 2015;21:998-1009.

10 Kang HM, Ahn SH, Choi P, Ko YA, Han SH, Chinga F, Park AS, Tao J, Sharma K, Pullman J, Bottinger EP, Goldberg IJ, Susztak K: Defective fatty acid oxidation in renal tubular epithelial cells has a key role in kidney fibrosis development. Nat Med 2015;21:37-46.

11 Wei Q Dong Z: Mouse model of ischemic acute kidney injury: technical notes and tricks. Am J Physiol Renal Physiol 2012;303:F1487-1494.

12 Available at http://cancan.cshl.edu/labmembers/gordon/fastq_illumina_filter/

13 Joshi NA, Fass JN: Sickle: A sliding-window, adaptive, quality-based trimming tool for FastQ files. 2011 Available at https://github.com/najoshi/sickle

14 Trapnell C, Pachter L, Salzberg SL: TopHat: discovering splice junctions with RNA-Seq. Bioinformatics 2009;25:1105-1111.

15 Li H, Handsaker B, Wysoker A, Fennell T, Ruan J, Homer N, Marth G, Abecasis G, Durbin R; 1000 Genome Project Data Processing Subgroup:The Sequence Alignment/Map format and SAMtools. Bioinformatics 2009;25:2078-2079.

16 Trapnell C, Williams BA, Pertea G, Mortazavi A, Kwan G, van Baren MJ, Salzberg SL, Wold BJ, Pachter L: Transcript assembly and quantification by RNA-Seq reveals unannotated transcripts and isoform switching during cell differentiation. Nat Biotechnol 2010;28:511-515.

17 Goff L, Trapnell C, Kelley D: cummeRbund: Analysis, exploration, manipulation, and visualization of Cufflinks high-throughput sequencing data.. R package version 2.14.0. 2013 Available at http://compbio. mit.edu/cummeRbund/

18 Kim JH, Karnovsky A, Mahavisno V, Weymouth T, Pande M, Dolinoy DC, Rozek LS, Sartor MA.: LRpath analysis reveals common pathways dysregulated via DNA methylation across cancer types. BMC Genomics 2012;13:526.

-19 Lee C, Patil S, Sartor MA: RNA-Enrich: a cut-off free functional enrichment testing method for RNA-seq with improved detection power. Bioinformatics 2016;32:1100-1102. 


\section{Cellular Physiology Cell Physiol Biochem 2018;47:1338-1351 \begin{tabular}{l|l} 
DOI: 10.1159/000490819 & $\begin{array}{l}\text { O 2018 The Author(s). Published by S. Karger AG, Basel } \\
\text { www.karger.com/cpb }\end{array}$
\end{tabular} \\ Bataille et al.: FAO in Injured Kidneys}

20 Supek F, Bošnjak M, Škunca N, Šmuc T: REVIGO summarizes and visualizes long lists of gene ontology terms. PLoS ONE 2011;6:e21800.

-21 Legouis D, Bataille A, Hertig A, Vandermeersch S, Simon N, Rondeau E, Galichon P: Ex vivo analysis of renal proximal tubular cells. BMC Cell Biol 2015;16:12.

-22 Portilla D, Dai G, Peters JM, Gonzalez FJ, Crew MD, Proia AD: Etomoxir-induced PPARalpha-modulated enzymes protect during acute renal failure. Am J Physiol Renal Physiol 2000;278:F667-675.

-23 Luo F, Shi J, Shi Q Xu X, Xia Y, He X: Mitogen-Activated Protein Kinases and Hypoxic/Ischemic Nephropathy. Cell Physiol Biochem 2016;39:1051-1067.

-24 Guan X, Qian Y, Shen Y, Zhang L, Du Y, Dai H, Qian J, Yan Y: Autophagy protects renal tubular cells against ischemia / reperfusion injury in a time-dependent manner. Cell Physiol Biochem 2015;36:285-298.

-25 Li Y, Zhong D, Lei L, Jia Y, Zhou H, Yang B: Propofol Prevents Renal Ischemia-Reperfusion Injury via Inhibiting the Oxidative Stress Pathways. Cell Physiol Biochem 2015;37:14-26.

26 Preising C, Schneider R, Bucher M, Gekle M, Sauvant C: Regulation of Expression of Renal Organic Anion Transporters OAT1 and OAT3 in a Model of Ischemia/Reperfusion Injury. Cell Physiol Biochem 2015;37:113.

27 Brandoni A, Torres AM: Altered Renal Expression of Relevant Clinical Drug Transporters in Different Models of Acute Uremia in Rats. Role of Urea Levels. Cell Physiol Biochem 2015;36:907-916.

28 Sánchez-Calvo B, Cassina A, Rios N, Peluffo G, Boggia J, Radi R, Rubbo H, Trostchansky A: Nitro-Arachidonic Acid Prevents Angiotensin II-Induced Mitochondrial Dysfunction in a Cell Line of Kidney Proximal Tubular Cells. PLoS ONE 2016;11:e0150459.

29 Bin Feng, Meng R, Bin Huang, Bi Y, Shen S, Zhu D: Silymarin protects against renal injury through normalization of lipid metabolism and mitochondrial biogenesis in high fat-fed mice. Free Radic Biol Med 2017;110:240-249. 